\title{
Altered functional connectivity in seizure onset zones revealed by $\mathrm{fMRI}$ intrinsic connectivity OPEN
}

Hyang Woon Lee, MD, $\mathrm{PhD}$

Jagriti Arora, MS

Xenophon Papademetris, $\mathrm{PhD}$

Fuyuze Tokoglu, MS Michiro Negishi, PhD

Dustin Scheinost, MS

Pue Farooque, MD

Hal Blumenfeld, MD, $\mathrm{PhD}$

Dennis D. Spencer, MD R. Todd Constable, PhD

Correspondence to

Dr. Lee:

leeh@ewha.ac.kr

Supplemental data at Neurology.org

\section{ABSTRACT}

Objective: The purpose of this study was to investigate functional connectivity (FC) changes in epileptogenic networks in intractable partial epilepsy obtained from resting-state fMRI by using intrinsic connectivity contrast (ICC), a voxel-based network measure of degree that reflects the number of connections to each voxel.

Methods: We measured differences between intrahemispheric- and interhemispheric-ICC

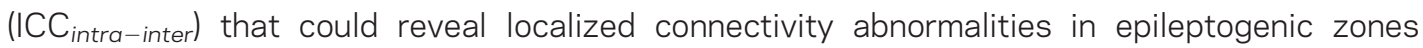
while more global network changes would be eliminated when subtracting these values. The ICC intra-inter map was compared with the seizure onset zone (SOZ) based on intracranial EEG (icEEG) recordings in 29 patients with at least 1 year of postsurgical follow-up. Two independent reviewers blindly interpreted the icEEG and fMRI data, and the concordance rates were compared for various clinical factors.

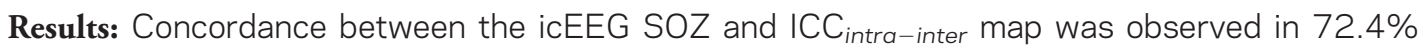
(21/29) of the patients, which was higher in patients with good surgical outcome, especially in those patients with temporal lobe epilepsy (TLE) or lateral temporal seizure localization. Concordance was also better in the extratemporal lobe epilepsy than the TLE group. In 85.7\% (18/21) of the cases, the ICC intra-inter values were negative in the SOZ, indicating decreased FC within the epileptic hemisphere relative to between hemispheres.

Conclusions: Assessing alterations in FC using FMRI-ICC map can help localize the SOZ, which has potential as a noninvasive presurgical diagnostic tool to improve surgical outcome. In addition, the method reveals that, in focal epilepsy, both intrahemispheric- and interhemispheric-FC may be altered, in the presence of both regional as well as global network abnormalities. Neurology ${ }^{\circledR}$ 2014;83:2269-2277

\section{GLOSSARY}

$\mathbf{C I}=$ confidence interval; $\mathbf{E T L E}=$ extratemporal lobe epilepsy; $\mathbf{E Z}=$ epileptogenic zone; $\mathbf{F C}=$ functional connectivity; $\mathbf{F o V}=$ field of view; ICC = intrinsic connectivity contrast; icEEG = intracranial EEG; $\mathbf{M N I}=$ Montreal Neurological Institute; MPRAGE = magnetization-prepared rapid acquisition with a gradient echo; rs-fMRI = resting-state fMRI; SOZ = seizure onset zone; TE $=$ echo time; TLE $=$ temporal lobe epilepsy; TR $=$ repetition time.

Epilepsy can be conceptualized as a network disorder that may involve not only epileptogenic regions but also remote cortical areas. ${ }^{1,2}$ Examination of epileptogenic networks may provide a better understanding of seizure generation and propagation to other brain regions. Furthermore, understanding the network properties may help guide surgical intervention for better postoperative outcomes in refractory epilepsy. Various measures of connectivity have been used to better understand epileptogenic networks. ${ }^{2-6}$

Functional connectivity (FC) measurements from resting-state fMRI (rs-fMRI) data can provide new insights into epileptogenic networks. ${ }^{7}$ Previous studies in focal epilepsy, focusing mostly on medial temporal lobe epilepsy (TLE), reported variable results: FC usually decreased

From the Department of Neurology (H.W.L.), Ewha Womans University School of Medicine and Ewha Medical Research Institute, Seoul, Korea; and Departments of Neurology (H.W.L., P.F., H.B.), Diagnostic Radiology (H.W.L., J.A., X.P., F.T., M.N., R.T.C.), Biomedical Engineering (X.P., D.S., R.T.C.), Neurosurgery (H.B., D.D.S., R.T.C.), and Neurobiology (H.B.), Yale University School of Medicine, New Haven, CT. Go to Neurology.org for full disclosures. Funding information and disclosures deemed relevant by the authors, if any, are provided at the end of the article. The Article Processing Charge was paid by internal funds in the Department of Diagnostic Radiology, Yale University School of Medicine. This is an open access article distributed under the terms of the Creative Commons Attribution-Noncommercial No Derivative 3.0 License, which permits downloading and sharing the work provided it is properly cited. The work cannot be changed in any way or used commercially. 
but sometimes increased in the seizure focus, and also changed in the contralateral temporal and other cortical areas. ${ }^{8-16}$ Examining FC changes might help localize the epileptogenic zone (EZ).${ }^{17}$ Nevertheless, few rs-fMRI studies have compared this directly with postsurgical outcome.

To apply fMRI FC analysis to localize the EZ regardless of the seizure focus requires a voxel-based analysis method that does not rely on a priori assumptions as to specific regions of interest. Epileptogenic networks may involve nodes ipsilateral or contralateral to the seizure focus, thus network properties could lead to both intrahemispheric- and interhemisphericFC alterations. ${ }^{7}$ To distinguish the seizure focus from other changes in remote brain areas, a contrast between intrahemisphericand interhemispheric-FC was introduced. Differences between intrahemispheric- and interhemispheric-FC maps were compared with the seizure onset zone (SOZ) identified based on intracranial EEG (icEEG) recordings in epilepsy surgery patients. We also compared the concordance rates in subgroups of various clinical factors.

METHODS Subjects. We analyzed data retrospectively from 29 patients with intractable partial epilepsy. The inclusion criteria were patients who (1) were referred for anatomical MRI and rs-fMRI between October 2004 and February 2011, (2) subsequently underwent epilepsy surgery after icEEG seizure recordings, and (3) had at least 1 year of postsurgical follow-up. The patients' data were compared with those of 85 normal healthy volunteers $($ male/female $=44: 41$, mean age $34.7 \pm$ 11.3 years)

Standard protocol approvals, registrations, and patient consents. Written informed consent was obtained from all participants. This study was approved by the Human Investigational Committee of the Yale University School of Medicine.

Data acquisition. All patients had 2 to 8 runs of rs-fMRI using a 3T MR scanner (Siemens Trio, Siemens Medical, Erlangen, Germany) or a $1.5 \mathrm{~T}$ MR scanner (Siemens Sonata) with short breaks between runs (less than a minute) to provide high-quality data free of motion artifacts. Images were acquired with an echoplanar imaging sequence with a 2 -second repetition time (TR), 30-millisecond echo time (TE), 256-mm field of view (FoV), $90^{\circ}$ flip angle, $64 \times 64$ matrix size, $4 \times 4 \mathrm{~mm}$ in-plane resolution, and 4-mm slice thickness without gap, generating 220 image volumes. Each control subject had 8 runs of rs-fMRI using the $3 \mathrm{~T}$ MR scanner with the same imaging parameters.

Anatomical images were acquired with 2-dimensional (300-millisecond TR, 2.43-millisecond TE, $60^{\circ}$ flip angle) and 3-dimensional T1-weighted sagittal magnetization-prepared rapid acquisition with a gradient echo (MPRAGE) sequence (2,530-millisecond TR, 3.34-millisecond TE, $7^{\circ}$ flip angle) with 256-mm FoV, $256 \times 256$ matrix size, 1 -mm thickness, and 176 contiguous slices.

FC analysis using intrinsic connectivity contrast. To calculate the intrinsic connectivity contrast-degree (ICC- $\mathrm{d}_{t h}$ ) measure, the correlation between the rs-fMRI BOLD time course of a reference voxel and all other gray matter voxels was determined, and the number of such correlations above a correlation threshold $(>0.25)$ was used as a measure of the degree reflecting network connectivity. ${ }^{18}$

First, 2 ICC- $\mathrm{d}_{t h}$ maps were computed for each voxel to capture hemispheric differences in FC after preprocessing. The first map was of a voxel's connectivity within the ipsilateral hemisphere $\left(\mathrm{ICC}_{\text {intra }}\right.$. The second map was of voxel's connectivity to the contralateral hemisphere $\left(\mathrm{ICC}_{\text {inter }}\right)$. Both maps were generated for each voxel in the gray matter. Finally, the difference map was created by subtracting ICC $_{\text {inter }}$ from ICC intra $_{\text {(ICC }}$ intra-inter $)$ to cancel out global network alterations (figure 1). ICC values were normalized to fit a gaussian distribution with zero mean and unitary variance, and smoothed with a 6-mm gaussian kernel. To compute the group statistics, single subject results were normalized to the Montreal Neurological Institute (MNI) standard template using the intensity-only component as implemented in BioImage Suite software (www.bioimagesuite.org). ${ }^{19,20}$

Differences between each individual patient and the 85 control subjects were identified using a group $t$ test $(p<0.05$ with family-wise error correction, 20-voxel cluster threshold). The ICC $_{\text {intra-inter }}$ map was initially created with a threshold $t$ value of 2.0 in each patient, then adjusted stepwise by a $t$ value of 0.05 to a higher threshold if the map showed diffuse and widespread changes, or to a lower threshold if it revealed too subtle changes. Regions with reliable ICC differences were identified anatomically by using the Talairach Daemon Atlas ${ }^{21}$ after transforming MNI coordinates into Talairach coordinates corrected using a nonlinear transformation. ${ }^{22}$

Anatomical localization of 3-dimensional electrode positions and coregistration to ICC maps. Intracranial electrodes (Ad-Tech Medical Instruments Corp., Racine, WI) were implanted as part of surgery procedures. All patients underwent pre- and postoperative MRI and CT scans with subdural electrodes. MRI was performed as described above, and Lightspeed CT (Siemens Medical) was done with parameters of $120 \mathrm{kVp}, 600$ milliseconds $/ 220 \mathrm{~mA}$, helical/64 mode, $1.5-\mathrm{mm}$ thickness without gap, and $260 \times 220 \mathrm{~mm} \mathrm{FoV}$. Electrode locations were identified using the postoperative CT and postoperative MRI, and registered to the preoperative $M R I,{ }^{23}$ then coregistered to the MPRAGE image of individual patients using BioImage Suite. ${ }^{19}$ This sequence of registrations allows the electrode locations and ICC maps to be coregistered and displayed on the individual's MPRAGE image (figure 1).

Identification of the icEEG SOZ. All patients underwent icEEG recording using 128-channel video-EEG monitoring equipment (Bio-Logic Systems Corp., Mundelein, IL) with 16-bit A/D conversion, 256- $\mathrm{Hz}$ sampling, $90-\mathrm{dB}$ common mode rejection ratio, and $0.1-$ to $\sim 70-\mathrm{Hz}$ bandpass filter. First, the icEEG was reviewed separately, blind to other clinical information, by 2 expert neurologists (H.W.L. and P.F.) to identify the $\mathrm{SOZ}$ using traditional icEEG visual analysis. If the results disagreed, the 2 reviewers discussed the case to reach a final consensus or declare the case nonlocalizing if they could not localize the $\mathrm{SOZ}$ or failed to reach an agreement.

Concordance between the icEEG SOZ and AMRI-ICC maps. The concordance rate was evaluated between the 
Figure 1 Diagram summarizing the preprocessing steps and the creation of the ICC map to compare with the icEEG SOZ

\section{icEEG electrode maps}
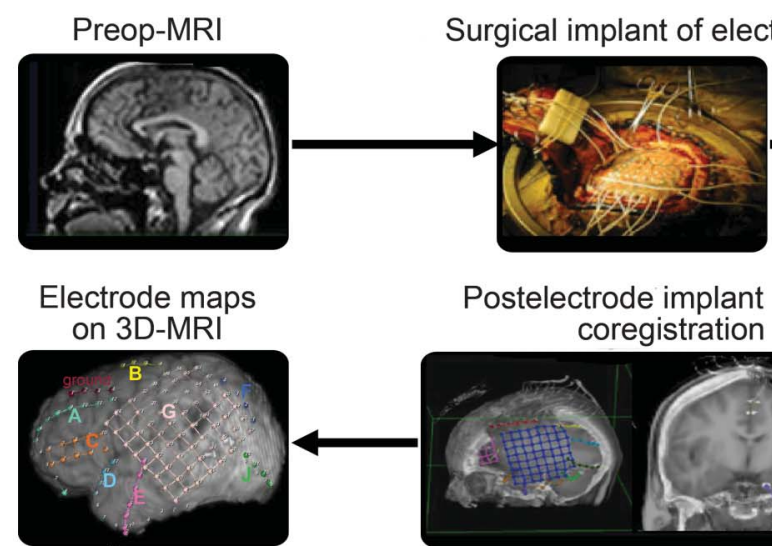

Postelectrode implant CT-MRI coregistration

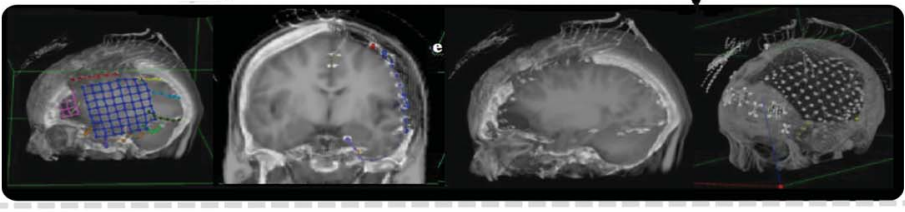

icEEG seizure onset

Seizure onset

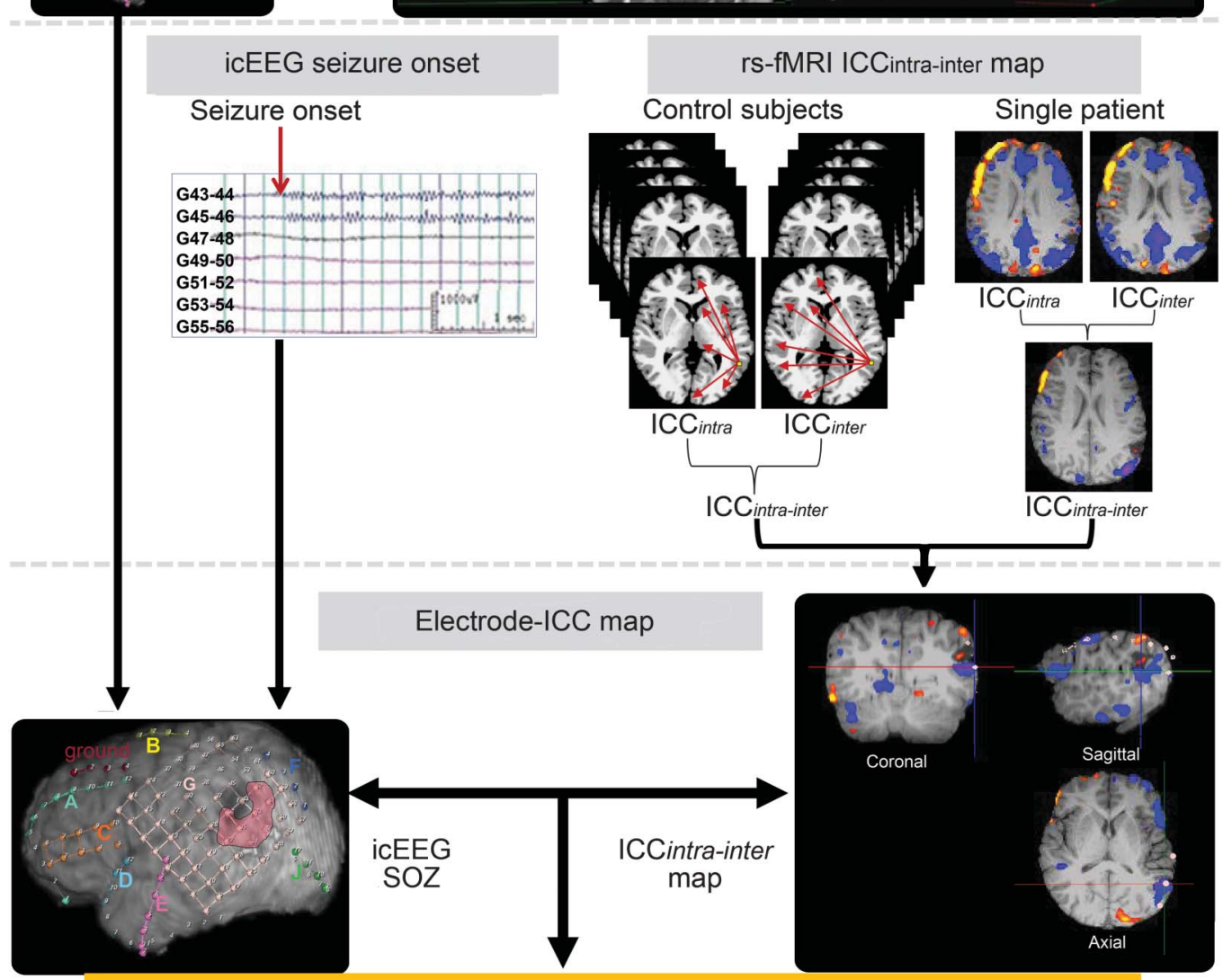

rs-fMRI ICCintra-inter map

Comparison between icEEG SOZ vs fMRI ICC map

The ICC was measured for each voxel, reflecting the number of connections to each voxel from all other voxels in the gray matter either within the same hemisphere (intrahemispheric) or between hemispheres (interhemispheric). After normalization of the ICC maps with those from control subjects, the within-subject difference of intrahemispheric- and interhemispheric-ICC (ICC intra-inter) was compared with the icEEG SOZ in individual patients. ICC = intrinsic connectivity contrast; icEEG = intracranial EEG; rs-fMRI = resting-state fMRI; SOZ = seizure onset zone.

fMRI-ICC map and icEEG SOZ, not resected cortical area in postoperative imaging. This was because not all of these patients had postoperative MRI (some of them had postoperative CT instead) and several patients had multiple subpial transections with or without partial surgical resection since the cortical area included a functionally eloquent region. The results of ICC maps were interpreted separately by 2 reviewers (H.W.L. and R.T.C.) who were blinded to all other clinical data. The fMRI-ICC maps were presented to the 2 reviewers separately, in random patient order. In cases with multiple cortical regions with mixed increased or decreased ICC changes, only the cortical area showing the largest cluster with the peak intensity difference was taken into account. In the same manner as for the icEEG, if the 2 reviewers disagreed, 
they discussed the case to reach a consensus or categorize it as nonlocalizing.

For both the icEEG SOZ and AMRI-ICC maps, the reviewers interpreted the cortical localization of abnormality as to the side, lobar, or sublobar. The concordance between these modalities was determined on the coregistered 3-dimensional brain surface rendering of electrode locations and ICC maps in each patient. Concordance was determined only when there was spatial overlap between the SOZ and ICC change regions at the same sublobar location. Surgical outcomes were determined based on the Engel classification $^{24}$ and International League Against Epilepsy outcome criteria. ${ }^{25}$

Statistical analysis. To assess the reliability of each test modality, agreement rates were calculated between the reviewers based on their initial independent interpretations using Cohen $\kappa$ coefficient. The reliability of concordance between the icEEG SOZ and ICC maps was determined based on the final consensus using generalized $\kappa$-type statistics. ${ }^{26}$ The $\chi^{2}$ test for proportions was used to compare concordance rates in subgroups of patients with various clinical factors including surgical outcome and final localization. Statistical results were calculated using Stata 10.0 (StataCorp, College Station, TX) for $\kappa$ coefficients and SPSS 16.0.0 (SPSS Inc., Chicago, IL) for other tests, with $p<0.05$ considered significant.

RESULTS The patients were 14 males and 15 females, with the age range of 7 to 55 years (mean $29.4 \pm 11.3$ ). There were 13 patients with TLE and 16 with extratemporal lobe epilepsy (ETLE), with a mean seizure onset age of $12.4 \pm 8.1$ years and a mean epilepsy duration of $13.9 \pm 7.4$ years (table 1). The interrater agreement rate for ICC $_{\text {intra- }}$ -inter interpretation was $89.7 \%(\kappa=0.66,95 \%$ confidence interval $[\mathrm{CI}]=0.31-1.00)$, which was comparable to that of the icEEG SOZ (agreement rate $93.1 \%, \kappa=0.79,95 \%$ CI $0.51-1.00)$. ICC $_{i n-}$ tra-inter maps showed localized changes in 23 of 29 patients. Among them, 21 patients showed concordance of their $\mathrm{ICC}_{\text {intra-inter }}$ maps with their icEEG SOZ $(91.3 \%, \kappa=0.76,95 \%$ CI $0.44-1.00)$.

In 18 of the cases with concordance (18/21, 85.7\%), $\mathrm{ICC}_{\text {intra-inter }}$ was negative in the SOZ, indicating decreased FC within the epileptic hemisphere relative to between hemispheres. The remaining 3 patients with concordance $(3 / 21,14.3 \%)$ had positive values of $\mathrm{ICC}_{\text {intra-inter }}$ in the $\mathrm{SOZ}$, indicating lower interhemispheric FC than intrahemispheric FC.

Representative cases are shown in figures 2 and 3. Patient 5 in table 1 with lesional TLE had negative

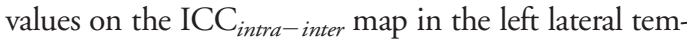
poral cortical region that matched well with the icEEG $\mathrm{SOZ}$ at the posteroinferior border of the structural lesion (figure 2), indicating lower connectivity within the epileptic hemisphere relative to between hemispheres in this area. In contrast, patient 14 with lesional ETLE had positive values on the ICC $_{\text {intra-inter }}$ map in the right parietooccipital SOZ (figure 3), indicating lower interhemispheric relative to intrahemispheric
FC in this area. These 2 patients were seizure free after surgery.

The overall concordance between the icEEG SOZ and $\mathrm{ICC}_{\text {intra-inter }}$ map was higher in patients with good surgical outcome than those with poor outcome $(93.3 \%$ vs $50.0 \%)$. This was particularly the case in patients with TLE (85.7\% vs $50.0 \%$ ) and lateral temporal seizure localization $(80.0 \%$ vs $0 \%)$. Concordance was also better in the ETLE than TLE group (93.8\% vs $\left.46.2 \%, \chi^{2}=8.134, p=0.010\right)$. Of note, concordance was good in ETLE regardless of seizure outcome (100\% vs $87.5 \%$ ), while in TLE, it was better with good outcome than poor outcome $(85.7 \%$ vs $0 \%)$ (table 2). No specific trends were observed in other patient subgroups (table 2, table e-1 on the Neurology ${ }^{\circledR}$ Web site at Neurology.org).

DISCUSSION This study demonstrates that FC mapping at the voxel level, based on rs-fMRI data, may provide additional information for the identification of the EZ, potentially improving surgical outcome. Differences between intrahemispheric- and interhemispheric-ICC values ( ICC $_{\text {intra-inter }}$ ) were mapped and compared with the icEEG SOZ in 29 epilepsy surgery patients. The main findings of this study were as follows: (1) concordance between the icEEG SOZ and ICC $_{\text {intra-inter }}$ map was observed in $72.4 \%$ (21/29), (2) ICC $_{\text {intra-inter }}$ maps in the SOZ showed lower FC within the epileptic hemisphere relative to between hemispheres in $85.7 \%(18 / 21)$, and (3) concordance was higher in patients with good surgical outcome (especially in those with TLE and lateral temporal seizure localization) and also better in the ETLE than TLE group. Our findings suggest the possibility that this approach could add another information dimension in the workup of patients who are candidates for epilepsy surgery.

Most rs-fMRI studies in partial epilepsy, mainly targeting medial TLE, have reported decreased FC, ${ }^{6,8,9,14,16}$ but a few studies have shown increases in connectivity. ${ }^{11,17}$ In addition to changes in the seizure focus, contralateral temporal or remote cortical changes were reported as well. , $, 12,13,15,16$ The variability of these findings might be related to patient heterogeneity or methodologic differences. Only a few patients with neocortical epilepsy (included mainly in the TLE cohorts) have been studied with rs-fMRI so far. ${ }^{4,17}$ Changes in areas remote to the seizure focus or in the contralateral hemisphere have also been reported in other human studies using different neuroimaging modalities such as PET. ${ }^{27,28}$ The mechanisms for changes outside the seizure foci are not completely understood, but frequent seizure spread with comorbid neurobehavioral abnormalities and possible compensatory mechanisms have been suggested. ${ }^{29} \mathrm{~A}$ study using a tetanus toxin rat model 
Table 1 Clinical information of all patients and concordance between intracranial EEG seizure onset and fMRI-ICC maps

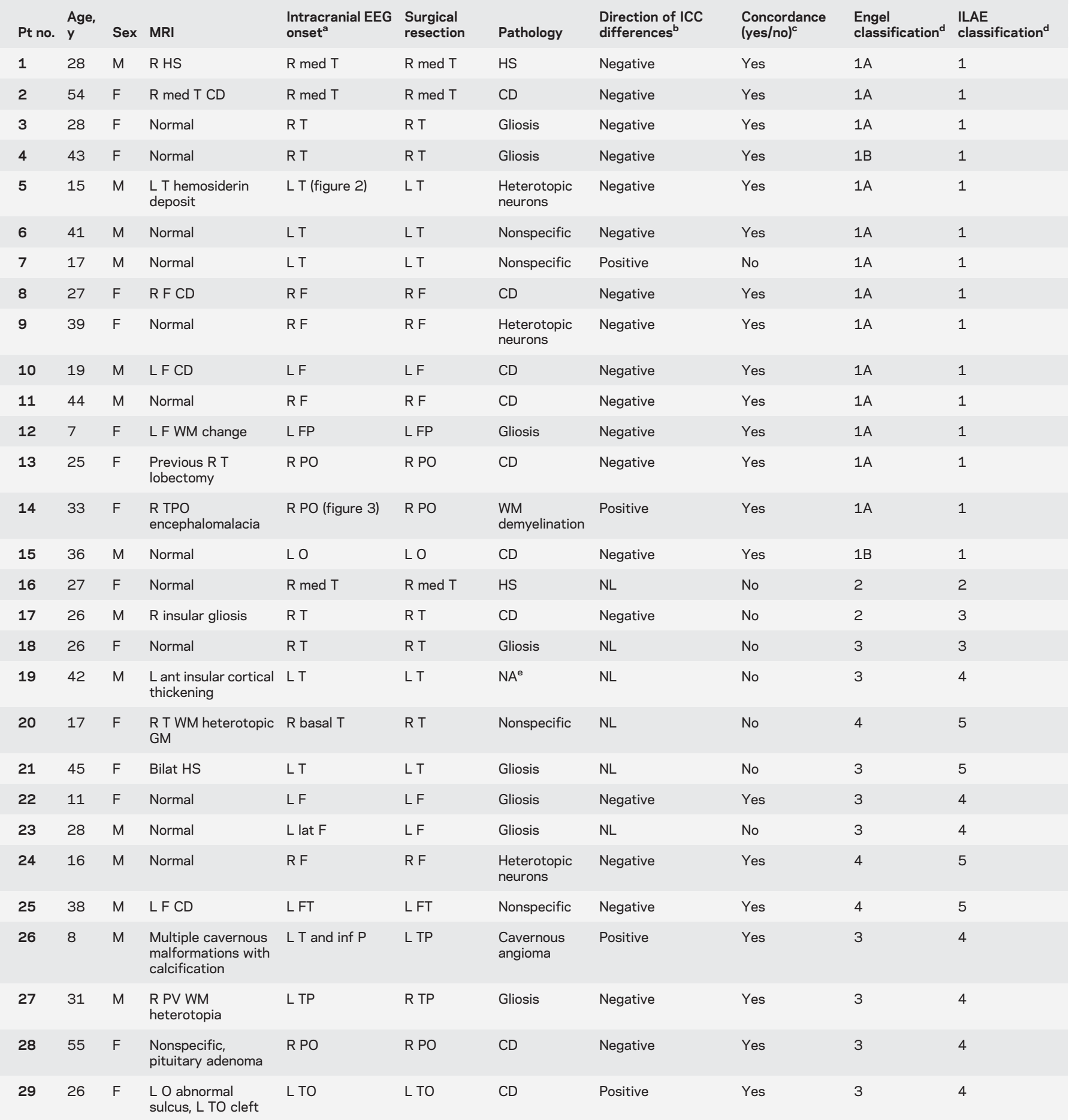

Abbreviations: ant $=$ anterior; bilat $=$ bilateral; $\mathrm{CD}=$ cortical dysplasia; $\mathrm{F}=$ frontal; $F \mathrm{FP}=$ frontoparietal; $\mathrm{FT}=$ frontotemporal; $\mathrm{GM}=$ gray matter; hemi $=$ hemisphere; HS = hippocampal sclerosis; ICC = intrinsic connectivity contrast; ILAE = International League Against Epilepsy; inf = inferior; lat = lateral; med $=$ medial; $\mathrm{NA}=$ not available; $\mathrm{NL}=$ nonlocalized; $\mathrm{O}=$ occipital $\mathrm{P}=$ parietal; $\mathrm{PO}=$ parietooccipital; $\mathrm{pt}=$ patient; $\mathrm{PV}=$ periventricular; sup $=$ superior; $\mathrm{T}=$ temporal; $\mathrm{TO}=$ temporooccipital; TP $=$ temporoparietal; TPO = temporoparieto-occipital; $\mathrm{WM}=$ white matter.

${ }^{\text {a } B a s e d ~ o n ~ i n t r a c r a n i a l ~ s e i z u r e ~ o n s e t ~ e l e c t r o d e s . ~}$

${ }^{\mathrm{b}}$ Either negative or positive difference in $\mathrm{ICC}_{\text {intra-inter }}$ maps.

${ }^{\mathrm{c}}$ Either concordant (yes) or discordant (no) based on the sublobar localization.

dPostsurgical seizure outcome.

e Pathology not available because the patient performed multiple subpial transections instead of surgical resection. 

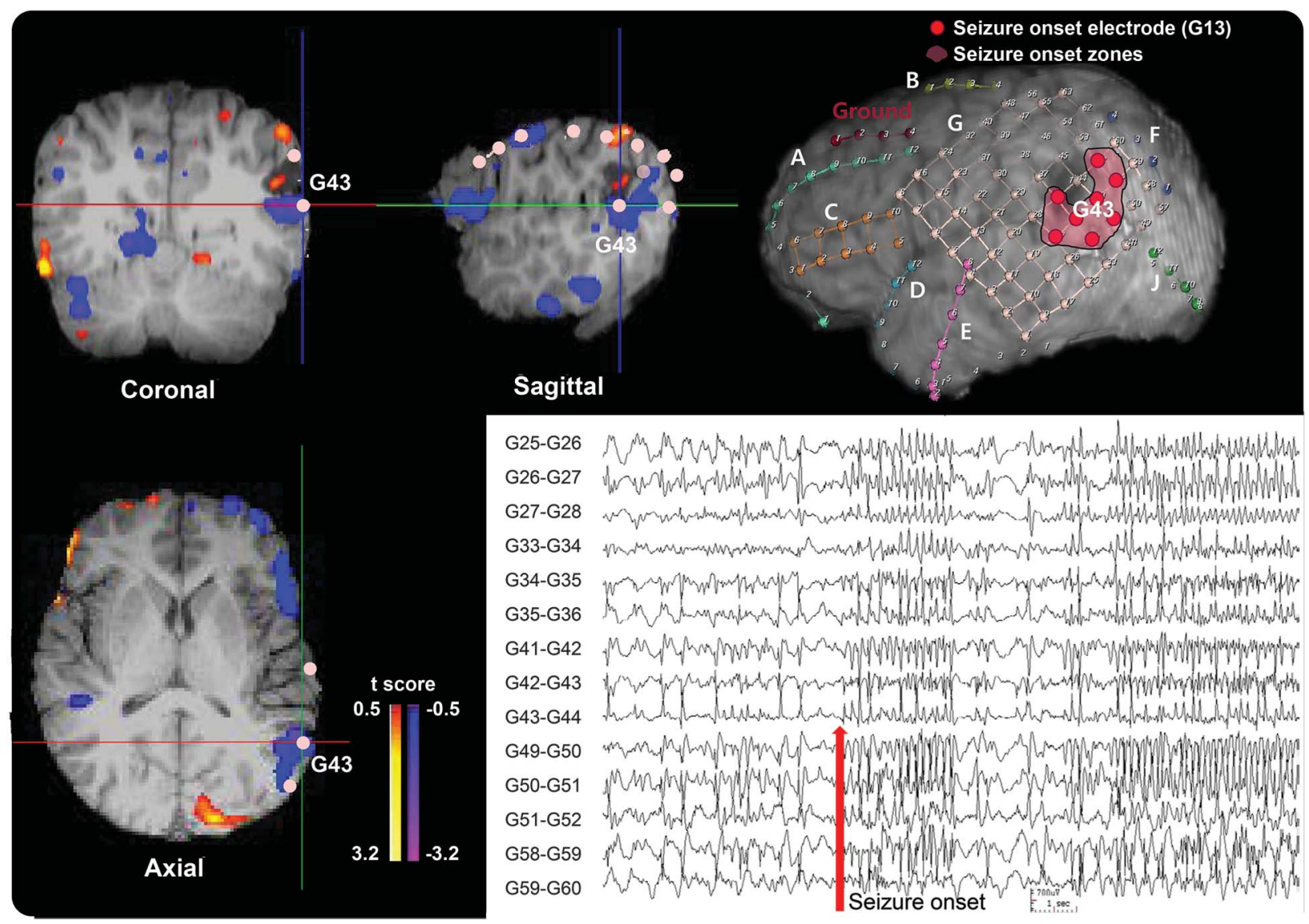

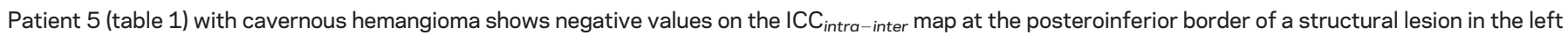

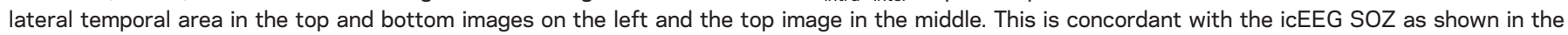

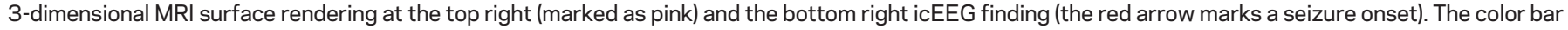

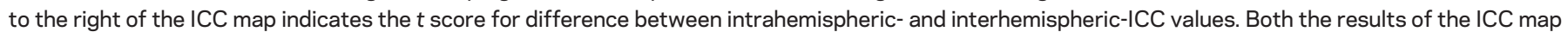

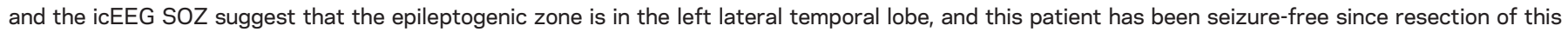
region. ICC = intrinsic connectivity contrast; icEEG = intracranial EEG; SOZ = seizure onset zone.

for focal neocortical epilepsy, where the etiology and the seizure focus were controlled to be identical in all animals, showed decreased interhemispheric but increased intrahemispheric FC in the epileptic and nonepileptic hemispheres, but these changes altered with time. ${ }^{30}$ In this focal epilepsy model, the functional alterations observed were not restricted to the epileptic focus but were in multiple brain regions, supporting the possibility that both regional and global changes occur in neocortical epilepsy.

It is somewhat counterintuitive that the location of the SOZ stands out when subtracting the interhemispheric-ICC from intrahemispheric-ICC in the majority of our patients. This finding suggests that $\mathrm{FC}$ changes in the EZ have characteristic features that are distinct within the epileptic hemisphere or between hemispheres, while FC outside the epileptic foci is relatively stable within the epileptic and the contralateral hemispheres. FC could be influenced in different ways when comparing local vs remote connectivity (e.g., the former is more related to the epileptogenesis itself while the latter is associated with more global changes in network properties).

Recent evidence has suggested that a laterality index based on FC could be helpful to predict surgical outcomes but this does not provide information on the EZ. ${ }^{4}$ In the current study, we examined whether FC changes could have localizing value in epilepsy surgery patients. The concordance between the icEEG SOZ and AMRI FC changes was analyzed to investigate whether these FC changes truly represent the EZ. We observed that the concordance rate between the 2 modalities was higher in patients with good surgical outcome. Of note, concordance was good in ETLE regardless of surgical outcome, while in TLE it was higher in patients with good outcome. These findings suggest that this kind of FC mapping approach may be helpful in improving seizure localization for subgroups of patients with otherwise poor outcome.

Various methods of network analysis based on graph theory have been used to assess FC in epileptic 


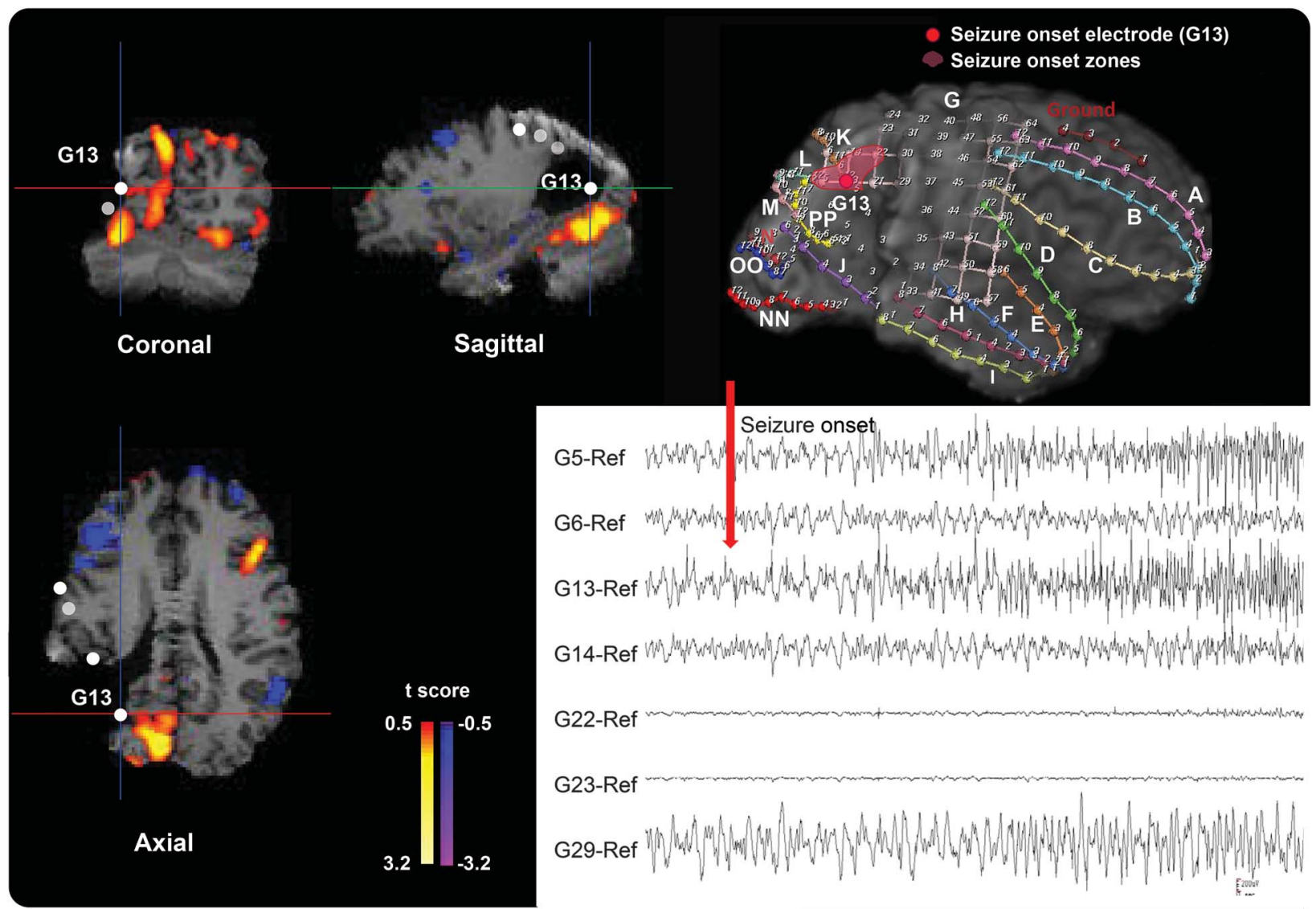

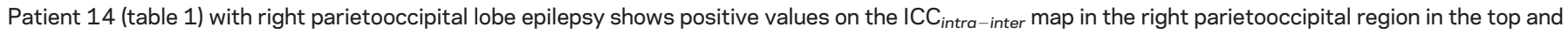
bottom images on the left and the top image in the middle. This matches well with the icEEG SOZ shown in the 3-dimensional MRI surface rendering at the top right (marked as pink) and the bottom right icEEG finding (the red arrow marks a seizure onset). The color bar to the right of the ICC map indicates the $t$ score for difference between intrahemispheric- and interhemispheric-ICC values. Both the results of the ICC map and the icEEG SOZ suggest that the epileptogenic zone is in the right parietooccipital lobe, and this patient has been seizure-free since resection of this region. ICC $=$ intrinsic connectivity contrast; icEEG = intracranial EEG; SOZ = seizure onset zone.

networks. ${ }^{3,6-17,31,32}$ A recent study revealed increased FC in the icEEG SOZ when using local FC measures within close cortical areas. ${ }^{17}$ In the current analysis, we used the $\mathrm{ICC}_{\text {intra-inter }}$ map, since this highlighted localized changes while the individual $\mathrm{ICC}_{\text {intra }}$ and ICC $_{\text {inter }}$ maps showed diffuse and bilateral changes that were insufficient for localization in most cases. Healthy normal brain tissue typically demonstrates more within hemisphere connectivity and less contralateral hemisphere connectivity. ${ }^{7}$ In contrast, the negative values of $\mathrm{ICC}_{\text {intra-inter }}$ in the $\mathrm{EZ}$ in most of our patients may indicate less FC in that region. Traditionally, the interictal epileptiform discharges observed on EEG are characterized as increased synchrony in the EZ. ${ }^{33,34}$ Previous studies using scalp or icEEG reported increased connectivity within the seizure focus, but decreased connectivity outside of the focus in TLE ${ }^{11,35}$ and ETLE. ${ }^{36}$ Several other studies have challenged this classic view showing that the synchrony and/or correlation of EEG activity are dynamically changing over time, or even decreased at seizure onset. ${ }^{37}$ Our study suggests that the epileptic hemisphere exhibits lower connectivity than the contralateral side, although that does not indicate absolute changes. To assess that, future studies in larger and more homogeneous groups of patients would be needed.

One of the difficulties in localizing the ICC changes was that the ICC maps often showed multifocal changes in cortical areas in addition to those areas that matched the SOZ. It is unclear at this time whether the multiple focal regions (1) result from an epileptogenic network rather than a single seizure focus, (2) reflect other aspects of functional changes such as comorbid neurobehavioral abnormalities, ${ }^{31,38}$ (3) relate to artifacts that require further technical improvement in analysis methods, or (4) result from a combination of all of the above. The next logical step to fully consider all the possibilities would be to perform network analyses (using the same rs-fMRI data) on these multiple nodes to determine the central node and other network properties and to correlate these with detailed clinical data. 


\begin{tabular}{|c|c|c|c|c|c|c|}
\hline \multirow[t]{2}{*}{ Table 2} & \multicolumn{6}{|c|}{$\begin{array}{l}\text { Concordance rates between intracranial EEG seizure onset and fMRI-ICC maps in different clinical } \\
\text { conditions according to postsurgical seizure outcome }\end{array}$} \\
\hline & & Good outcome & Poor outcome & Total & $x^{2}$ & $p$ Value \\
\hline Overall ( $\mathrm{N}$ & 29) & 14/15 (93.3) & $7 / 14(50.0)$ & 21/29 (72.4) & 6.807 & $0.014^{a}$ \\
\hline \multicolumn{7}{|c|}{ Epileptic syndrome } \\
\hline \multicolumn{2}{|c|}{ TLE $(n=13)$} & 6/7 (85.7) & 0/6 (0.0) & 6/13 (46.2) & 9.551 & $0.005^{b}$ \\
\hline \multicolumn{2}{|c|}{ ETLE $(n=16)$} & $8 / 8(100.0)$ & $7 / 8$ (87.5) & 15/16 (93.8) & 1.067 & 1.000 \\
\hline \multicolumn{7}{|c|}{ MRI lesion } \\
\hline \multicolumn{2}{|c|}{ Nonlesional $(n=13)$} & $6 / 7$ (85.7) & $3 / 6(50.0)$ & 9/13 (69.2) & 1.935 & 0.266 \\
\hline \multicolumn{2}{|c|}{ Lesional $(n=16)$} & 8/8 (100.0) & $4 / 8(50.0)$ & $12 / 16(75.0)$ & 5.333 & 0.077 \\
\hline \multicolumn{7}{|c|}{ Lobar/sublobar localization } \\
\hline \multicolumn{2}{|c|}{ Medial temporal $(n=3)$} & $2 / 2(100.0)$ & 0/1 (0.0) & 2/3 (66.7) & 3.000 & 0.333 \\
\hline \multicolumn{2}{|c|}{ Lateral temporal $(n=10)$} & $4 / 5(80.0)$ & 0/5 (0.0) & $4 / 10(40.0)$ & 6.667 & $0.048^{a}$ \\
\hline \multicolumn{2}{|c|}{ Frontal $(n=7)$} & $4 / 4(100.0)$ & 2/3 (66.7) & $6 / 7$ (85.7) & 1.556 & 0.429 \\
\hline \multicolumn{2}{|c|}{ Occipital $(n=1)$} & $1 / 1(100.0)$ & None & $1 / 1(100.0)$ & - & - \\
\hline \multicolumn{2}{|c|}{$\mathrm{FT}(\mathrm{n}=1)$} & None & $1 / 1(100.0)$ & $1 / 1(100.0)$ & - & - \\
\hline \multicolumn{2}{|c|}{$\mathrm{FP}(\mathrm{n}=1)$} & $1 / 1(100.0)$ & None & $1 / 1(100.0)$ & - & - \\
\hline \multicolumn{2}{|c|}{$\mathrm{TP}(\mathrm{n}=2)$} & None & 2/2 (100) & $2 / 2(100.0)$ & - & - \\
\hline \multicolumn{2}{|c|}{$\mathrm{PO}(\mathrm{n}=3)$} & 2/2 (100) & $1 / 1(100.0)$ & 3/3 (100.0) & - & - \\
\hline \multicolumn{2}{|c|}{ TO $(n=1)$} & None & $1 / 1(100.0)$ & $1 / 1(100.0)$ & - & - \\
\hline
\end{tabular}

Abbreviations: $E T L E$ = extratemporal lobe epilepsy; FP = frontoparietal; FT = frontotemporal; ICC = intrinsic connectivity contrast; $\mathrm{PO}=$ parietooccipital; TLE $=$ temporal lobe epilepsy; $\mathrm{TO}=$ temporooccipital; $\mathrm{TP}=$ temporoparietal.

Data are $\mathrm{n}(\%)$.

${ }^{a} p<0.05$ and ${ }^{b} p<0.01$ with statistical significance.

However, the overall validity of this method is still under investigation and warrants further studies to develop an automated and quantifiable method to select significant connectivity clusters of interest.

FC measurement using rs-fMRI has the potential to provide a novel noninvasive method to localize the $\mathrm{SOZ}$ as a part of presurgical evaluation. In our study, the concordance rate between the icEEG SOZ and fMRI-ICC map was better in patients with good outcome, suggesting that this kind of approach can be useful as a biomarker that reflects epileptogenesis and also predicts surgical outcome in advance when planning epilepsy surgery. For instance, patients in whom brain areas showing ICC changes are resected completely would be expected to have better surgical outcome. Although it is still in the early stage of development, with further validation in patients with intractable partial epilepsy, this kind of approach may ultimately be useful for both clinical and scientific purposes, especially as part of presurgical evaluations. We hope that our study represents a step forward in developing clinically useful analysis paradigms using fMRI-based intrinsic connectivity measurements.

\section{AUTHOR CONTRIBUTIONS}

Hyang Woon Lee: design and conceptualization of the study design, subject selection, analysis and interpretation of the data, statistical analysis, drafting and revising the manuscript. Jagriti Arora: data collection, analysis of the fMRI data, statistical analysis, and revising the manuscript. Xenophon Papademetris: analysis of the fMRI data and revising the manuscript. Fuyuze Tokoglu: data collection and analysis of the fMRI data. Michiro Negishi: data collection and analysis of the fMRI data. Dustin Scheinost: analysis of the fMRI data and revising the manuscript. Pue Farooque: analysis, interpretation of the icEEG data, and revising the manuscript. Hal Blumenfeld: subject selection, data collection, interpretation of the icEEG data, and revising the manuscript. Dennis D. Spencer: data collection, analysis of the icEEG data, and revising the manuscript. R. Todd Constable: study design, interpretation of the fMRI data, and revising the manuscript.

\section{STUDY FUNDING}

Dr. Lee is funded by the Basic Science Research Program through the National Research Foundation (NRF) of Korea by the Ministry of Science, ICT, and future planning (NRF-2011-0015788 and 2014-R1A2A1A11052103), and by the Ewha Global Top 5 Grant 2011 of Ewha Womans University of Korea. Dr. Blumenfeld is funded by the NIH grant NS055829. Dr. Constable is funded by the NIH grant EB009666-02.

\section{DISCLOSURE}

The authors report no disclosures relevant to the manuscript. Go to Neurology.org for full disclosures.

Received May 31, 2013. Accepted in final form September 11, 2014.

\section{REFERENCES}

1. Kramer MA, Cash SS. Epilepsy as a disorder of cortical network organization. Neuroscientist 2012;18:360-372.

2. Lemieux L, Daunizeau J, Walker MC. Concepts of connectivity and human epileptic activity. Front Syst Neurosci 2011;5:1-13. 
3. Bettus G, Ranjeva JP, Wendling F, et al. Interictal functional connectivity of human epileptic networks assessed by intracerebral EEG and BOLD signal fluctuations. PLoS One 2011;6:e20071.

4. Negishi M, Martuzzi R, Novotny EJ, Spencer DD, Constable RT. Functional MRI connectivity as a predictor of the surgical outcome of epilepsy. Epilepsia 2011;52: $1733-1740$

5. Wilke C, Worrell GA, He B. Analysis of epileptogenic network properties during ictal activity. Conf Proc IEEE Eng Med Biol Soc 2009;2009:2220-2223.

6. Zhang X, Tokoglu F, Negishi M, et al. Social network theory applied to resting-state fMRI connectivity data in the identification of epilepsy networks with iterative feature selection. J Neurosci Methods 2011;199:129-139.

7. Constable RT, Scheinost D, Finn ES, et al. Potential use and challenges of functional connectivity mapping in intractable epilepsy. Front Neurol 2013;4:39. doi: 10.3389/fneur.2013.00039.

8. Bettus G, Guedj E, Joyeux F, et al. Decreased basal fMRI functional connectivity in epileptogenic networks and contralateral compensatory mechanisms. Hum Brain Mapp 2009;30:1580-1591.

9. Bettus G, Bartolomei F, Confort-Gouny S, et al. Role of resting state functional connectivity MRI in presurgical investigation of mesial temporal lobe epilepsy. J Neurol Neurosurg Psychiatry 2010;81:1147-1154.

10. Frings L, Schulze-Bonhage A, Spreer J, Wagner K. Reduced interhemispheric hippocampal BOLD signal coupling related to early epilepsy onset. Seizure 2009;18:153-157.

11. Liao W, Zhang Z, Pan Z, et al. Altered functional connectivity and small-world in mesial temporal lobe epilepsy. PLoS One 2010;5:e8525.

12. Morgan VL, Gore JC, Abou-Khalil B. Cluster analysis detection of functional MRI activity in temporal lobe epilepsy. Epilepsy Res 2007;76:22-33.

13. Morgan VL, Gore JC, Abou-Khalil B. Functional epileptic network in left mesial temporal lobe epilepsy detected using resting fMRI. Epilepsy Res 2010;88:168-178.

14. Pereira FR, Alessio A, Sercheli MS, et al. Asymmetrical hippocampal connectivity in mesial temporal lobe epilepsy: evidence from resting state fMRI. BMC Neurosci 2010;11:66.

15. Zhang Z, Lu G, Zhong Y, et al. Impaired perceptual networks in temporal lobe epilepsy revealed by resting fMRI J Neurol 2009;256:1705-1713.

16. Zhang Z, Lu G, Zhong Y, et al. fMRI study of mesial temporal lobe epilepsy using amplitude of low-frequency fluctuation analysis. Hum Brain Mapp 2010;31:1851-1861.

17. Stufflebeam SM, Liu H, Sepulcre J, Tanaka N, Buckner RL, Madsen JR. Localization of focal epileptic discharges using functional connectivity magnetic resonance imaging. J Neurosurg 2011;114:1693-1697.

18. Martuzzi R, Ramani R, Qiu M, Shen X, Papademetris X, Constable RT. A whole-brain voxel based measure of intrinsic connectivity contrast reveals local changes in tissue connectivity with anesthetic without a priori assumptions on thresholds or regions of interest. Neuroimage 2011;58:1044-1050.

19. Joshi A, Scheinost D, Okuda H, et al. Unified framework for development, deployment and robust testing of neuroimaging algorithms. Neuroinformatics 2011;9:69-84.
20. Papademetris X, Jackowski AP, Schultz RT, Staib LH, Duncan JS. Integrated intensity, point-feature nonrigid registration. Med Image Comput Comput Assist Interv 2001;3216:763-770.

21. Lancaster JL, Woldorff MG, Parsons LM, et al. Automated Talairach atlas labels for functional brain mapping. Hum Brain Mapp 2000;10:120-131.

22. Lacadie CM, Fulbright RK, Rajeevan N, Constable RT, Papademetris X. More accurate Talairach coordinates for neuroimaging using non-linear registration. Neuroimage 2008;42:717-725.

23. Meltzer JA, Zaveri HP, Goncharova II, et al. Effects of working memory load on oscillatory power in human intracranial EEG. Cereb Cortex 2008;18:1843-1855.

24. Engel J Jr, van Ness PC, Rasmussen TB, Ojemann LM. Outcome with respect to epileptic seizures. In: Engel J Jr, editor. Surgical Treatment of the Epilepsies, 2nd ed. New York: Raven Press; 1993:609-622.

25. Wieser HG, Blume WT, Fish D, et al. ILAE Commission Report: proposal for a new classification of outcome with respect to epileptic seizures following epilepsy surgery. Epilepsia 2001;42:282-286.

26. Landis JR, Koch GG. The measurement of observer agreement for categorical data. Biometrics 1977;33:159-174.

27. Bouilleret V, Dupont S, Spelle L, Baulac M, Samson Y, Semah F. Insular cortex involvement in mesiotemporal lobe epilepsy: a positron emission tomography study. Ann Neurol 2002;51:202-208.

28. Chassoux F, Semah F, Bouilleret V, et al. Metabolic changes and electro-clinical patterns in mesio-temporal lobe epilepsy: a correlative study. Brain 2004;127:164-174.

29. Koepp MJ, Woermann FG. Imaging structure and function in refractory focal epilepsy. Lancet Neurol 2005;4: 42-53.

30. Otte WM, Dijkhuizen RM, van Meer MP, et al. Characterization of functional and structural integrity in experimental focal epilepsy: reduced network efficiency coincides with white matter changes. PLoS One 2012;7:e39078.

31. Holmes M, Folley BS, Sonmezturk HH, et al. Resting state functional connectivity of the hippocampus associated with neurocognitive function in left temporal lobe epilepsy. Hum Brain Mapp 2014;35:735-744.

32. Holmes MJ, Yang X, Landman BA, et al. Functional networks in temporal-lobe epilepsy: a voxel-wise study of resting-state functional connectivity and gray-matter concentration. Brain Connect 2013;3:22-30.

33. Dichter MA, Ayala GF. Cellular mechanism of epilepsy: a status report. Science 1987;237:157-164.

34. Kandel ER, Schwartz JH, Jessell TM. Principles of Neural Science, 4th ed. New York: McGraw-Hill; 2000.

35. Bettus G, Wendling F, Guye M, et al. Enhanced EEG functional connectivity in mesial temporal lobe epilepsy. Epilepsy Res 2008;81:58-68.

36. Shevon CA, Cappell J, Emerson R, et al. Cortical abnormalities in epilepsy revealed by local EEG synchrony. Neuroimage 2007;35:140-148.

37. Schindler K, Leung H, Elger CE, Lehnertz K. Assessing seizure dynamics by analyzing the correlation structure of multichannel intracranial EEG. Brain 2007;130:65-77.

38. Vlooswijk MC, Vaessen MJ, Jansen JF, et al. Loss of network efficiency associated with cognitive decline in chronic epilepsy. Neurology 2011;77:938-944. 


\section{Neurology}

\section{Altered functional connectivity in seizure onset zones revealed by fMRI intrinsic connectivity}

Hyang Woon Lee, Jagriti Arora, Xenophon Papademetris, et al. Neurology 2014;83;2269-2277 Published Online before print November 12, 2014

DOI 10.1212/WNL.0000000000001068

This information is current as of November 12, 2014

\section{Updated Information \&} Services

Supplementary Material

\section{References}

Subspecialty Collections

Permissions \& Licensing

Reprints including high resolution figures, can be found at: http://n.neurology.org/content/83/24/2269.full

Supplementary material can be found at: http://n.neurology.org/content/suppl/2014/11/12/WNL.0000000000001 068.DC1

This article cites 36 articles, 3 of which you can access for free at: http://n.neurology.org/content/83/24/2269.full\#ref-list-1

This article, along with others on similar topics, appears in the following collection(s):

Epileptogenic zone

http://n.neurology.org/cgi/collection/epileptogenic_zone

fMRI

http://n.neurology.org/cgi/collection/fmri

Intracranial electrodes

http://n.neurology.org/cgi/collection/intracranial_electrodes

Information about reproducing this article in parts (figures,tables) or in its entirety can be found online at:

http://www.neurology.org/about/about_the_journal\#permissions

Information about ordering reprints can be found online:

http://n.neurology.org/subscribers/advertise

Neurology ${ }^{\circledR}$ is the official journal of the American Academy of Neurology. Published continuously since 1951, it is now a weekly with 48 issues per year. Copyright () 2014 American Academy of Neurology. All rights reserved. Print ISSN: 0028-3878. Online ISSN: 1526-632X.

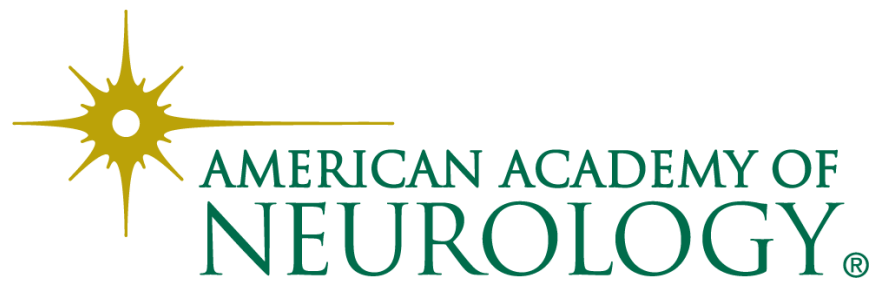

